\title{
Routine duodenal biopsies to diagnose celiac disease
}

\author{
Marietta lacucci MD PhD, Subrata Ghosh FRCP FRCPE FRCPC FCAHS, Editor-in-Chief
}

$\mathrm{C}$ eliac disease is increasingly detected by family physicians or gastroenterologists using immunoglobulin A antitissue transglutaminase antibody serology, which has operating characteristics perfectly suited for a screening test. Whether a biopsy is still required to confirm the diagnosis is debated, but in clinical practice, an endoscopy and biopsy is often performed to confirm the diagnosis (1). The serological titres are then often followed-up to assess adherence to gluten-free diet and adequate resolution of celiac disease by diet.

Upper endoscopy is a common procedure performed by gastroenterologists to investigate dyspepsia, dysphagia and other upper gastrointestinal symptoms. This offers an opportunity to obtain duodenal biopsies to establish or exclude the diagnosis of celiac disease. However, this approach requires considerable investment of time and resources by pathologists.

An important question, therefore, is whether duodenal biopsies should be routinely obtained at the time of endoscopy. Celiac disease may be associated with a multitude of symptoms and presentations such as bloating, diarrhea, abdominal discomfort, fatigue, weight loss, iron deficiency anemia, bone disease, skin disorders and abnormal liver function tests. Patients may also be completely asymptomatic but, of course, such patients generally do not present for upper endoscopy. While the prevalence of celiac disease continues to increase globally, a significant proportion of celiac disease patients remain undiagnosed. In the current issue of the Canadian Journal of Gastroenterology, Freeman (2) (pages 409-413) reports a long experience of diagnosing celiac disease using duodenal biopsies during upper endoscopy and the resultant increased diagnosis of celiac disease in the population. Duodenal bulb biopsies may increase diagnostic yield because villous atrophy may be patchy (3). A recent study has shown that modern high-definition endoscopic techniques with image enhancement may be very accurate in diagnosing villous atrophy (4). It may, therefore, be possible to only perform confirmatory duodenal biopsies, two from the duodenal bulb and four to six from the distal duodenum, where villous atrophy is suspected at high-definition endoscopy, with image enhancement techniques built into modern endoscopes. Marsh modified type 1 histology grade may, of course, miss recognition by endoscopic techniques. Endoscopic and histological demonstration of villous atrophy alone need to be distinguished from other causes of villous atrophy.

Therefore, when a patient is referred for upper endoscopy to confirm the diagnosis of celiac disease (such as in patients with positive celiac serology) or exclude celiac disease in patients with suspected celiac disease, it is, of course, mandatory to obtain biopsies from a second part of the duodenum and from the duodenal bulb. In patients referred for endoscopy to investigate upper gastrointestinal symptoms, but not suspected to have celiac disease, it may be acceptable to inspect the duodenum with high-definition endoscopy and imageenhancement techniques, and obtain biopsies if villous atrophy is

\section{REFERENCES}

1. Rubio-Tapia A, Hill ID, Kelly CP, Calderwood AH, Murray JA. ACG Clinical guidelines: Diagnosis and management of celiac disease. Am J Gastroenterol 2013;108:656-76.

2. Freeman H. Detection of adult celiac disease using duodenal screeing biopsies over a 30-year period. Can J Gastroenterol 2013;27:409-13.

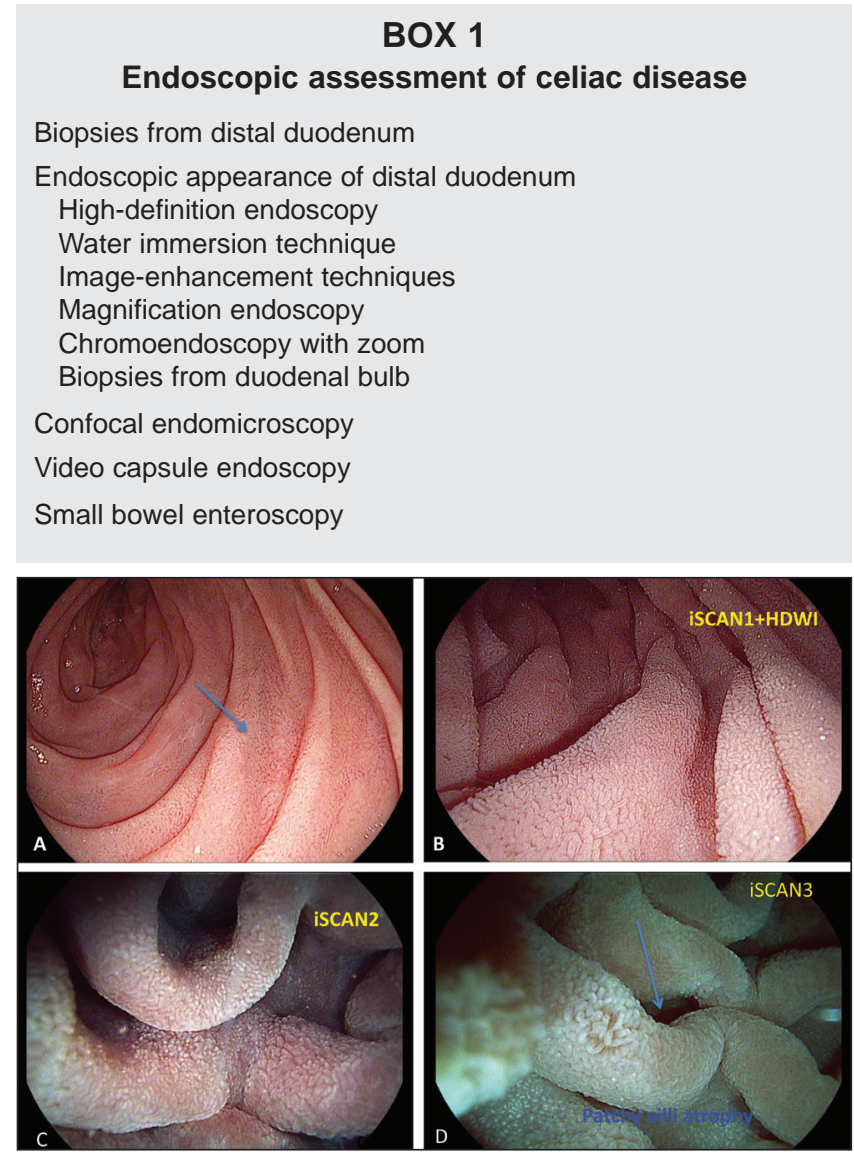

Figure 1) High-definition iScan (Pentax, Japan) technique showed patchy irregularity of the mucosa in the second portion of the duodenum. A Normal white-light endoscopy showing normal second part of duodenum. B to D iScan virtual chromoendoscopy highdefinition in combination with water immersion technique with virtual chromoendoscopy showing transitional patchy area of normal and atrophic villi. Biopsies confirmed celiac disease

detected (Box 1, Figure 1). However, standard white-light endoscopy alone will not be able to assess duodenal villous architecture. If biopsies at endoscopy confirm the diagnosis of celiac disease in patients who did not have celiac serology already performed, it will be essential to determine immunoglobulin $\mathrm{A}$ antitissue transglutaminase titres.

3. Kurien M, Evans KE, Hopper AD, Hale MF, Cross SS, Sanders DS. Duodenal bulb biopsies for diagnosing adult celiac disease: Is there an optimal biopsy site? Gastrointest Endosc 2012;75:1190-6.

4. Poon T, Gui S, Love JR, Ghosh S, Iacucci M. High-definition iSCAN endoscopy with water immersion technique accurately reflects histological severity of celiac disease. Digestive Diseases Week, Orlando, Florida, May 18 to 21, 2013. Oral Presentation 964.

Department of Medicine, University of Calgary, Calgary, Alberta

Correspondence: Dr Subrata Ghosh, Department of Medicine, Division of Gastroenterology, University of Calgary, 3280 Hospital Drive

Northwest, Calgary, Alberta T2N 4N1. Telephone 403-944-8222, fax 403-944-1095, e-mail subrata.ghosh@albertahealthservices.ca Received and accepted for publication June 7, 2013 


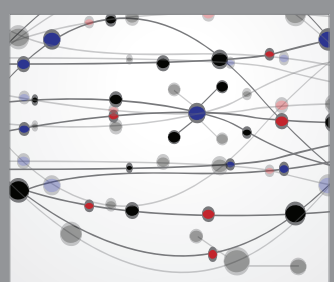

The Scientific World Journal
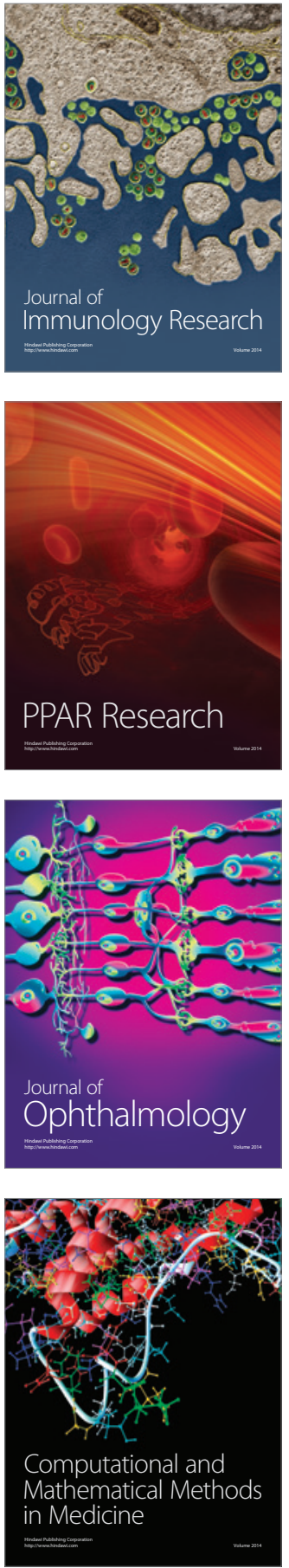

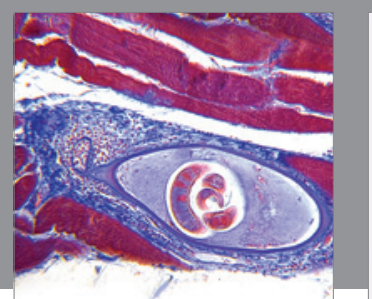

Gastroenterology Research and Practice

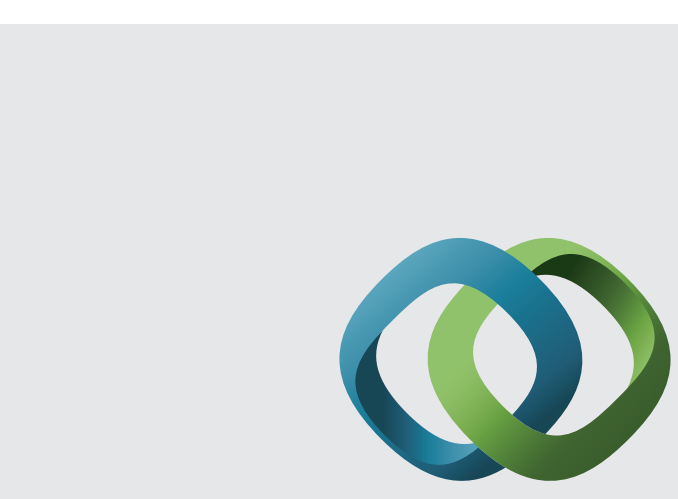

\section{Hindawi}

Submit your manuscripts at

http://www.hindawi.com
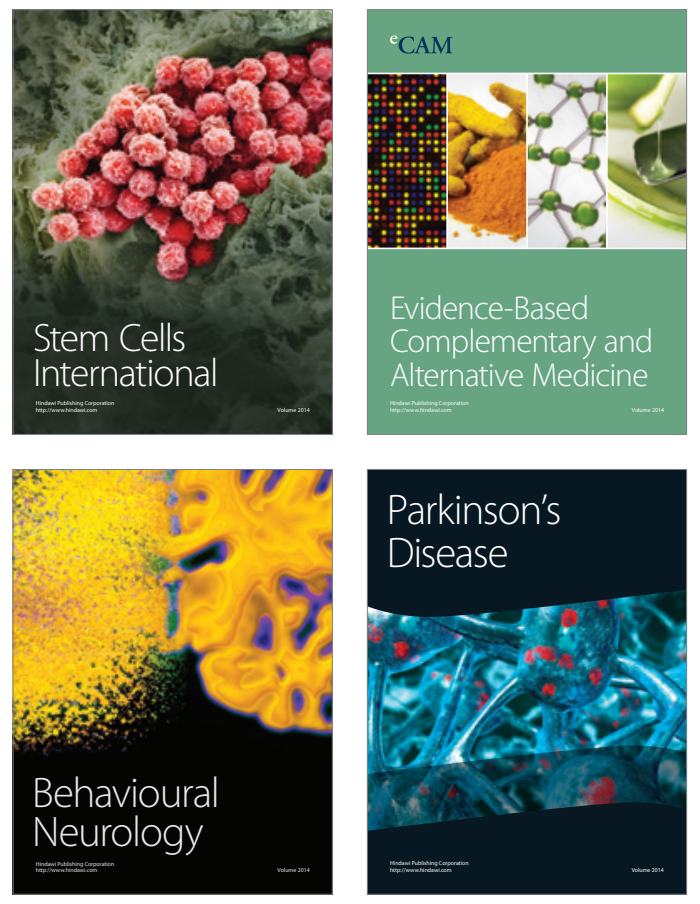
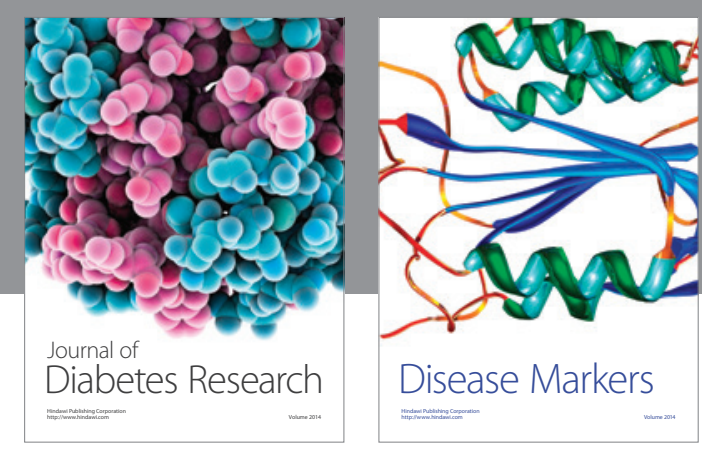

Disease Markers
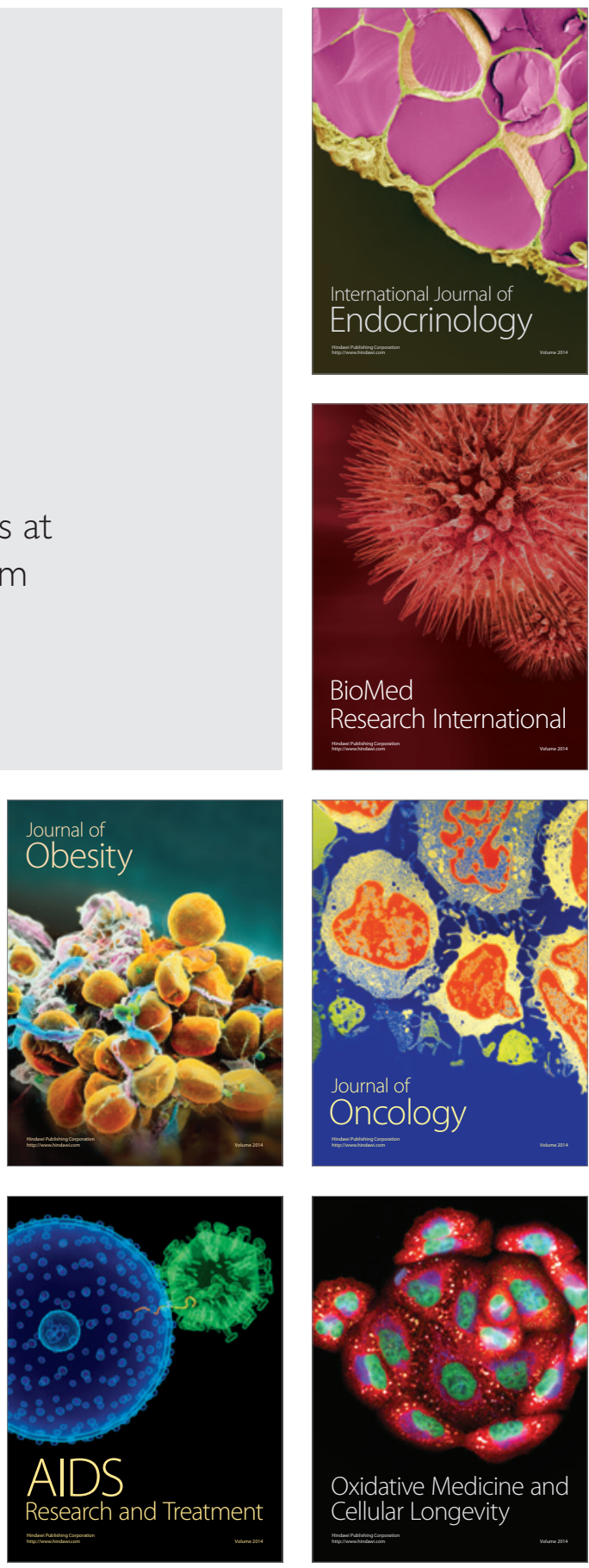